\title{
Convenciones en contabilidad desde la perspectiva de Jhon Searle*
}

DOI: http://dx.doi.org/10.21830/19006586.179

Recibido: 22 de febrero de 2017 • Aceptado: 5 de junio de 2017

\section{Conventions in Accounting from the Perspective of John Searle}

Des conventions en comptabilité dans la perspective de John Searle

Convenções em contabilidade a partir da perspectiva de John Searle

\author{
Edgar Gracia López ${ }^{a}$
}

* Este artículo es producto del proceso de investigación que se adelanta en el Grupo de Investigación de Teoría Contable de la Universidad de Manizales y forma parte integral del proceso de tesis que también adelanta el autor en la Universidad de los Andes de Venezuela con el doctorado en Ciencias Contables de esta institución.

a Universidad de los Andes, Mérida, Venezuela. Ph. D.(c) en Ciencias Contables, Universidad de los Andes de Venezuela.<egracia45@hotmai.com>. 
Resumen. En general, la contabilidad se encuentra ante la necesidad de validar sus condiciones teóricas y, en especial, de soportar las explicaciones acerca de la realidad que dice observar y analizar. Desde luego, en estos asuntos las complicaciones no se dejan esperar, máxime cuando son diversas las vertientes y posiciones de la contabilidad elaboradas en el curso de su desarrollo. En estas, se han depurado diferentes aproximaciones sobre la realidad, las cuales se constituyen en referentes para elucidar los contenidos y naturaleza de esta disciplina del conocimiento. De tal manera, el examen de la contabilidad desde las posiciones del filósofo Jhon R. Searle se constituye en un referente especial para seguir con el propósito de decantar explicaciones y comprensiones sobre las razones que asume esta disciplina del conocimiento, en cuanto al significado y realidad de los hechos, las instituciones, las convenciones y la función. En especial, el examen de la "función" en contabilidad brinda elementos importantes para comprender la relación de convención de la contabilidad con el contexto de la sociedad y las organizaciones.

Palabras clave: contabilidad; convención; función; institución; realidad contable.

Abstrac. Generally, accounting is faced to the need to validate its theoretical preconditions, in particular to ground the explanations about the reality it is told to observe and analyze. Certainly, as far these issues, complications may not be left alone, the more so when the starting points and contributions concerning accounting are very diverse, in the scope of their ongoing development. Among them, different approaches to reality has been chosen as suitable, so that they constitute references for elucidating contents and nature of this area of knowledge. Consequently, analyzing accounting from the perspective of the philosopher John Searle is really a special referent, in order to continue with the aim of depurating explanations and understandings on the reasons assumed by this area of knowledge, regarding the meaning and reality of facts, institutions, conventions, and its function. Especially, the inquiry of the "function" in accounting seems to give some key elements for understanding the relation of the accounting convention with the social and organizational context.

Key words: accounting; accounting reality; convention; function; institution.

Résumé. En général, la comptabilité s'efforce de valider ses conditions théoriques, notamment sur les motifs de appuyer ses explications sur la réalité qu'elle dit observer et analyser. C'est certain que dans ces questions, les difficultés ne se font pas attendre, surtout lorsque les positions adoptées et les propositions faite sont sur la comptabilité sont diversifiées, au cours de son déroulement. À cet égard, des approches différentes sur la réalité ont été retenu, de manière que ces aspects constituent de bonnes références pour clarifier le contenu et la nature de ce secteur de la connaissance. Par conséquent, l'analyse de la comptabiltité selon la pensée du philosophe John R. Searle est vraiment très spécial, afin de continue dans le but de comprendre des explications qui contiennent des éléments compliqués sur la rationalité assumée dans cet domaine, quant à la signification et la réalité des faits, les institutions, les conventions et sa function. Même s'il semble que l'examen de la «fonction » en comptabilité offre notamment des éléments importants pour comprendre la relation de la " convention» en comptabilité avec le contexte social et organisationnel.

Mots-clés: comptabilité; convention; function; institution; réalité comptable.

Resumo. Em geral, a contabilidade se confronta com a necessidade de validar as suas condições teóricas e especialmente apoiar as explicaçóes sobre a realidade que diz observar e analisar. Claro que nesses assuntos, surgem complicaçóes, especialmente quando as posiçôes desenvolvidas pela contabilidade são variadas no curso de seu desenvolvimento. Neste contexto, diferentes abordagens foram aperfeiçoadas ao longo realidade, que constituem referências para elucidar o conteúdo e a natureza da disciplina de conhecimento. Assim, o exame das contas das posiçóes do filósofo John R. Searle constitui uma referência especial para seguir a fim de decantar explicaçôes e insights sobre as razóes que assume a disciplina de conhecimento 
quanto ao significado e a realidade dos fatos, instituições, convenções e função. Especialmente o exame da "função" no fornecimento de contabilidade parece importante compreender a relação da convençâo contábil com o contexto da sociedade e das organizaçóes.

Palavras chave: contabilidade; convenção; função; instituição; realidade contabilidade.

\section{Introducción}

El objetivo de este escrito consiste en reflexionar sobre el sentido de las convenciones manifiestas en los órdenes teóricos y prácticos de la contabilidad. Para el efecto, se consideran los planteamientos de Searle (1997) relacionados en su obra Construcción de la realidad social, en la cual plantea la importancia de comprender la existencia y naturaleza de los hechos institucionales y de las funciones.

En estos aspectos, los criterios de Searle permiten aclaraciones sobre el sentido del lenguaje y la función para concretar la referencia a los denominados hechos. La importancia de este autor, precisamente, tiene que ver con la posibilidad que ofrece su trabajo para comprender las funciones que cumple la contabilidad en relación con las organizaciones, la sociedad y el campo de conocimiento.

Según Searle (1997), la pregunta central versa en últimas sobre la realidad, en el sentido de “¿cuál es la perspectiva concreta, la metodología concreta que permita describir la estructura de la realidad social?” (p. 25). Las respuestas otorgadas por este autor son relevantes para la contabilidad porque permiten reflexiones sobre la dupla ontológico-epistemológico y objetivo-subjetivo.

Como una manera de contrastar las apreciaciones de J. Searle (1997) se consideran las versiones de Berger y Luckmann (2001), autores que también intentan dar respuesta sobre lo que se refiere como realidad social y realidad objetiva. En últimas, en este artículo se trata de aclarar lo que se considera como realidad contable, aspecto que influye a la hora de elaborar las teorías y las explicaciones de la disciplina. En la actualidad, precisamente se cuestionan las formas como la contabilidad interpreta, evalú, mide e informa sobre los hechos que son parte de su campo de observación, de allí la importancia de entregar respuestas sobre la concepción y el recorte de la realidad que estudia la contabilidad.

\section{Sobre la realidad}

Inicialmente, es interesante mencionar dos sentidos que generan tensiones en la reflexión sobre las respuestas a las preguntas sobre la realidad: en Searle (1997), el contenido de "la construcción de la realidad social” insinúa una entrada metodológica que sugiere la preeminencia del sujeto en su sentido individual, mientras que en el caso de Berger y Luckmann (2001) el sentido de "la construcción social de la realidad" sugiere una entrada que destaca una instancia de comprensión colectiva y social como base para dar respuestas sobre la realidad considerada.

En la versión del filósofo J. Searle (1997) se enuncia una especie de psicologismo, en el cual el sujeto otorga una carga valorativa que incide en la construcción de la realidad. En este plano es posible endilgarle a este autor una postura en relación con el individualismo metodológico que incluso 
él mismo parece criticar cuando afirma: "Las exigencias del individualismo metodológico parecen forzarnos a rechazar la intencionalidad colectiva y a aceptar la intencionalidad individual" (p. 43).

En la versión de Berger y Luckmann (2001), el examen de la realidad exige que al inicio de la reflexión se haga una consideración sobre la construcción social. De tal manera, los autores vislumbran una especial forma de contextualismo y evolucionismo social que demarca los criterios sobre lo concebido como realidad social. Para estos autores, el pensamiento individual aparece como consecuencia de lo social, con lo cual dan paso a una interpretación que se basa más en los criterios de la historia social que en atributos derivados de la personalidad de los sujetos. De tal manera, "la realidad se construye socialmente [...], es propia de los fenómenos como independientes de nuestra propia volición" (p. 13). En Berger y Luckmann (2001), "el interés sociológico en materia de 'realidad' y 'conocimiento' se justifica por el hecho de su relatividad social. Lo que es 'real' para un monje del Tíbet puede no ser 'real' para un hombre de negocios norteamericano" (p. 15).

Las consideraciones anteriores referidas a los sentidos de construcción de la realidad son de importancia para clarificar las explicaciones sobre lo que hace la contabilidad (ayuda a perfilar la interpretación objetiva-subjetiva). En general, la contabilidad convencional responde mejor al planteamiento de J. Searle (1997), pues, como tal, está incursa en una manera de interpretar la realidad a través de un marco metodológico apoyado más en el individualismo (economía neoclásica) y en criterios de oferta y demanda, en el cual los usuarios de la información son determinantes.

En otras palabras, decir que lo social es determinante de lo individual o, al contrario, lo individual determinante de lo social, tiene profundas implicaciones epistemológicas y metodológicas. En estos aspectos, por supuesto se encuentran incluidas explicaciones que han constituido tendencias: materialistas, subjetivistas, estructuralistas, neoestructuralistas, formalistas, dialécticas, entre otras, cada una con sus versiones sobre la realidad y el mundo.

Sin duda, en estos planos de explicar la realidad, las disciplinas trabajan arduamente, en especial las ciencias sociales, puesto que en ellas anida las consideraciones e interpretaciones de la interacción hombre-naturaleza-sociedad. La contabilidad, como parte de las ciencias sociales, igual trabaja por encontrar respuestas respecto de la consideración de los hechos que le competen en su campo de observación y estudio; de ello dan cuenta, entre otros, autores como Gaffikin (2006) y Laughlin (1999). Igual, autores como Habermas (1999) y Luhmann (1998), que elaboran sus cuerpos teóricos referidos desde disímiles y complejas consideraciones de la realidad, las cuales tienen que ver con los observadores, los sistemas y el mundo de la vida.

Para Jürgen Habermas (1999), en el sentido de la racionalidad, la realidad es una dialéctica de relación en la acción concebida por finalidades fundamentalmente políticas. De tal manera, la realidad de un saber viene a determinarse por su uso social y no en lo fundamental por su constitución interna. Para Luhmann (1998), en un sentido distinto, "la comunicación es la única garantía para la realidad del sistema social, no porque refleje o describa adecuadamente el mundo tal cual es [...], sino porque se le puede condicionar debido a su estado cerrado, exponiéndose a sí misma a pruebas de verificación" (p. 397).

Para Searle (2006), la construcción de la realidad es derivada de la intencionalidad colectiva, considerando que esta "es una noción muy general relativa a la direccionalidad de la mente [...], 
pero que necesitamos discutir el papel de la intencionalidad en los grupos sociales humanos" (p. 96), en especial la manera como constituyen los hechos.

La consideración postulada por J. Searle, en el sentido de que las acciones cotidianas engendran instituciones sociales únicamente apoyadas en la costumbre y el hábito, puede sustentar, por ejemplo, las explicativas convencionales de la contabilidad que por tradición se reconocen en la "general aceptación".

En buena parte de los desarrollos y explicaciones de la contabilidad, en especial del positivismo contable, se reconoce que estos han sido el resultado de la constitución de hechos derivados de la costumbre y del uso de prácticas adheridas a las dinámicas propias de las actividades económicas y comerciales. Precisamente, Hendriksen (1974) reconoce que "la condición más importante que diera origen a la evolución de la contabilidad fue el auge del comercio que se centró en las ciudades italianas medievales" (p. 27).

Puede incluso reconocerse que los lenguajes contables convencionales, nacidos especialmente de las prácticas comerciales y de los negocios, progresivamente sentaron las bases para el establecimiento de reglas (justas o injustas). Según Tua (1983), propiciaron la emergencia de lo que se conoce como subprograma de general aceptación, donde las "reglas de la contabilidad son el producto de la experiencia más que de la lógica. Con ello el subprograma de búsqueda se caracteriza porque el respaldo de una determinada norma radica en el hecho de ser comúnmente practicada" (p. 453). Para este autor:

El programa de investigación que se inicia con la general aceptación está claramente ligado a la aparición del capitalismo financiero y sus necesidades de información contable para un adecuado desarrollo del mercado de capitales. Su entramado conceptual de reglas contables responde fundamentalmente, aunque con alguna variación de unos casos a otros, a la teoría contractual pura de la sociedad mercantil, en la que la información contable se concibe como un medio de comunicación entre la empresa y su accionista o, en última instancia, entre aquella y sus inversores presentes o futuros. (1983, p. 454)

A partir de las costumbres comerciales y de los usos dados a la información contable, en la práctica se configuró una especial constitución de reglas definidas para la acción que tuvieron como fuente lo convencional, en el sentido del "debe hacerse porque es lo que se hace"; asunto que tempranamente colocó la convención y sus expresiones en entredicho respecto de las formulaciones del mundo científico y sus cánones de postulación y desarrollo. Por la vía de la aceptación general transformada en convención, los asuntos de la realidad basados en la costumbre y lo cotidiano terminaron, en la práctica, por reñir con los criterios de validez científica.

Por la vía de la aceptación general soportada en el mundo de las prácticas contables, asociadas a las actividades comerciales de los negocios, precisamente se inaugura la polémica sobre los usos de la contabilidad y sus fuentes de explicación. A esto se sumó que la general aceptación igual derivó en la ley de obligatorio cumplimiento.

La polémica parece centrarse entre las tensiones que suscitan la contabilidad observada desde la convención y aquella relacionada con el mundo de lo científico, exigente en lenguajes formales que especifican requisitos en términos de estructuras formales, lingüísticas y proposicionales. Este aspecto de las convenciones en contabilidad se puede comprender de mejor manera a partir de la 
Accounting Principle Board (APB), que trató todos los principios de contabilidad generalmente aceptados como convenciones porque, según Sunder (2005):

Estos llegaron a ser generalmente aceptados por acuerdo (a menudo tácito) más que por derivación formal de un conjunto de postulados o conceptos básicos. Los principios se desarrollaron con base en la experiencia, la razón, la costumbre, el uso y a una variable significativa, la necesidad práctica [...] incluyeron tres "convenciones modificadas": conservadurismo, énfasis en el ingreso y aplicación del criterio por la comunidad profesional de contables. (p. 222)

De hecho, por ejemplo, el conservadurismo en la contabilidad se explica a través de la prudencia como una convención que intenta proteger la empresa contra las implicaciones del futuro, en el reconocimiento de estimaciones futuras. En la práctica, se trata, como lo define Abbagnano (2007), de una manera incorporada para "actuar en forma justa y con cautela", es decir, la prudencia como convención que se asocia con "el comportamiento racional en todo campo o virtud que determina lo que es bueno o malo para el hombre" (p. 932), en este caso frente a contingencias económicas del futuro.

Desde este punto de vista, incluso, puede colegirse que las acciones cotidianas relacionadas con las actividades principalmente económicas, según Searle (1997), terminan por constituir hechos institucionales. En el caso de la contabilidad, constituyendo hechos por convención, respondiendo al uso del saber contable en relación con la necesidad de informar sobre actividades de comercio y negocio.

La observación de Hendriksen (1974) en relación con estos hechos convencionales y su explicación por la teoría de la contabilidad, es que como tales, es decir, como "hechos", no son medibles ni verificables independientemente, y, por consiguiente, no son realmente hechos. Antes bien, "son las relaciones económicas dentro del mundo de los negocios y conceptos, tales como 'valor' y 'utilidad' los que pueden aparecer de manera distinta a observadores diferentes" (p. 2).

\section{Hechos institucionales}

Una de las preguntas de J. Searle (1997) es ¿cómo construimos una realidad social objetiva? (p. 14). Para responder este interrogante, el autor se coloca en una vía ética diferente a la propuesta por el nihilismo epistemológico, que soslaya los valores de la objetividad epistémica, la claridad y el rigor empírico. Para el efecto, precisamente acude a las distinciones (nacidas de los sujetos) entre hechos brutos y hechos institucionales. Para conseguir este propósito de explicación, Searle defenderá una especie de filosofía sana centrada en el realismo y la concepción de verdad como correspondencia. Según Nicolás y Frápolli (2012), la formulación clásica de esta concepción de la verdad suele situarse en el famoso dictum de Aristóteles en Metafísica: "decir lo que es, que es, y de lo que no es, que no es, es verdadero" (p. 21).

Desde luego, respecto de la clasificación de los hechos en brutos e institucionales, a Searle se le objetará que como tales, todos los hechos son institucionales pues corresponden a una realidad socialmente construida. Igual se le objetará que al momento de hacer la descripción lingüística del 
hecho bruto se acude al lenguaje, que sin duda es un hecho institucional o construido. En este sentido, se le dirá que confunde la descripción lingüística del hecho bruto con el hecho bruto mismo.

Para Searle, los hechos brutos se distinguen de los institucionales, en especial por la función del desempeño. Se podría asegurar que aquello de lo cual no se ha determinado una función o ni siquiera se ha pensado, es simplemente un hecho bruto. En la distinción de Searle, precisamente lo institucional está atravesado por el lenguaje, el acuerdo y lo más importante, la función.

En otras palabras, los hechos brutos son aquellos que simplemente quedan encerrados en la materialidad que contienen. Aunque más allá, los hechos brutos son aquellos independientes de los deseos, creencias y necesidades cognitivas de los humanos. Al contrario, en palabras de Searle (2006), los hechos dependientes del observador son hechos institucionales. Al respecto afirma:

La mayoría de fenómenos que debatimos en economía, tales como el dinero, las instituciones financieras, las corporaciones, las transacciones comerciales y las ofertas públicas de acciones son todas dependientes del observador. Se puede decir que, en general, las ciencias naturales se preocupan de fenómenos independientes del observador y las ciencias sociales de fenómenos dependientes del observador. (p. 66)

Los hechos institucionales emergen a la consideración debido a la presencia del observador que construye la realidad social a partir del lenguaje y el acuerdo. Esto hace que los hechos institucionales lo sean dependientes de los acuerdos humanos. El dinero es el ejemplo más típico de hecho institucional. El dinero es un hecho institucional porque forma parte de los acuerdos sociales allegados para enfrentar las transacciones y la organización de los mercados. El dinero se trata como un hecho institucional porque sus características dependen del observador o de las actitudes de quienes resulten encerrados en su determinación.

El dinero u otras formas caracterizadas como hecho institucional (gobierno, familia, la propiedad, el matrimonio) forman parte de una realidad social creada por nosotros para nuestros propósitos. En ese sentido, como tal, el dinero es objeto de referencias epistemológicas objetivas-subjetivas y ontológicas objetivas-subjetivas (tabla 1).

Tabla 1. El dinero desde los referentes epistémico/ontológico y subjetivo/objetivo

\begin{tabular}{lll}
\hline \multicolumn{1}{c}{ Epistemológico } & \multicolumn{1}{c}{ Ontológico } \\
\hline Objetivo & $\begin{array}{l}\text { Asociaciones y vinculación que se } \\
\text { organizan en torno a intereses. }\end{array}$ & $\begin{array}{l}\text { El dinero en su color, forma, textura y } \\
\text { componentes del papel. }\end{array}$ \\
Subjetivo & $\begin{array}{l}\text { El dinero como estatus, prestigio } \\
\text { y poder. }\end{array}$ & $\begin{array}{l}\text { El dinero en su relación con el valor in- } \\
\text { dividual que se le otorgue; sentimientos. }\end{array}$ \\
\hline
\end{tabular}

Fuente: elaboración del autor derivada de las apreciaciones de Searle (1997).

Desde el punto de vista ontológico-objetivo, el dinero que circula es portador de un color, de una forma, textura, de componentes del papel que permiten percibirlo y establecer precisamente que se trata de dinero. En este caso ontológico-objetivo, se aborda la descripción de los componentes captados esencialmente por los sentidos. 
Desde el ángulo epistemológico-objetivo, el dinero puede verse como el sinfín de asociaciones que se organizan en torno a intereses; según Simmel (2010) "de este modo, se hace posible, primero, la pura objetividad en los propósitos de la asociación, su carácter puramente técnico, su pérdida de todo colorido personal" (p. 3). Desde lo ontológico-subjetivo, el dinero adquiere una valoración en relación con lo individual y asociada básicamente a sentimientos, mientras que el orden de lo epistemológico-subjetivo exigirá explicaciones relacionadas en su función con el poder, el prestigio, el estatus.

Para comprender la naturaleza de los hechos institucionales en la propuesta de Searle (1997), es pertinente señalar varios de los atributos que soportan su postura, entre estos:

1. Que la existencia de los hechos institucionales requiere de instituciones humanas (p. 21).

2. Que se requiere precisar los papeles funcionales de los objetos.

3. Que un hecho institucional no puede existir aislado, sino solo en un conjunto de relaciones sistemáticas con otros hechos (p. 52).

Respecto de los papeles funcionales de los objetos, la pregunta en cuestión es ¿para qué sirven los objetos? Por supuesto las respuestas solo corresponden a los sujetos con capacidad para realizar distinciones. En este caso, según Searle (1997), "las funciones nunca son intrínsecas, sino relativas al observador, así como el añadido de la asignación de función” (p. 24). Igual, "las funciones se asignan según los intereses de los usuarios y los observadores". Al respecto, de acuerdo con Searle (1997), la función solo puede ser cumplida "merced al acuerdo o a la aceptación colectiva, que es un elemento crucial en la creación de los hechos institucionales" (p. 56).

De acuerdo con Searle (1997), el tercer atributo para comprender la naturaleza de los hechos brutos e institucionales tiene que ver con su posición acerca de que "un hecho institucional no puede existir aislado, sino solo en un conjunto de relaciones sistemáticas con otros hechos" (p. 52). Desde este punto de vista, entonces, el papel de las funciones de los objetos, de la intencionalidad colectiva y de las reglas constitutivas se constituye en base para la construcción de realidad, siempre y cuando se tenga como referente la sociedad, el hecho social y, por supuesto, el conjunto de relaciones.

Siguiendo las apreciaciones de Searle (1997) en el caso de la contabilidad y respecto de su campo de observación, se puede afirmar que los hechos que retoma en su lógica de constitución son todos institucionales. Los hechos brutos para la contabilidad corresponderían a aquellos que por su naturaleza no tienen atributos para formar parte de su constitución como campo de observación. Por ejemplo, la psicología reconoce en su campo de estudio los fenómenos referidos a las patologías de la mente humana, asunto que no es del interés de la contabilidad, como sí lo son los fenómenos de la riqueza, la propiedad y el control, que como tales también son de referencia y observación para disciplinas del conocimiento como la economía, la sociología, la administración y el derecho. 


\section{Ontología fundamental}

En la posición de J. Searle (1997), la ontología social deriva de la ontología fundamental, desde donde funda las diversas ontologías de las cuales se pueden precisar atributos. Una ontología fundamental preexiste a una ontología social que "versa sobre el modo en que los hechos sociales existen” (p. 25), es decir, se trata de una ontología amplia. En este sentido, por ejemplo, la conciencia como realidad social derivaría de la física, en cuanto la conciencia es un rasgo biológico y, en consecuencia, físico. Según esta apreciación, para Searle (1997), "de la mano de la conciencia viene la intencionalidad, la capa de la mente para representar objetos y estados de cosas mundanos distintos de uno mismo" (p. 26).

En la ontología social los hechos sociales pueden ser observados como simples y complejos, a manera de una ontología simple o una ontología compleja (tabla 2). La ontología simple se refiere a la realidad creada en la relación de los objetos, dependiendo de la función, es decir, respondiendo a la pregunta ¿para qué sirven? Según Searle (1997), la ontología compleja identifica las cosas por sus rasgos intrínsecos sin hacer referencia a nuestros intereses (p. 24).

Tabla 2. Observación de los hechos en las duplas epistemológico/ontológico y objetivo/subjetivo

\begin{tabular}{cll}
\hline & \multicolumn{1}{c}{ Epistemológico } & \multicolumn{1}{c}{ Ontológico } \\
\hline \multirow{2}{*}{ Objetivo } & $\begin{array}{l}\text { Sentido basado en el juicio objetivo, indepen- } \\
\text { diente de las actitudes. }\end{array}$ & $\begin{array}{l}\text { Ontología compleja: rasgos intrínsecos de las co- } \\
\text { sas (paisajes, moléculas, montañas). Existencia } \\
\text { independiente de los sujetos. }\end{array}$ \\
\multirow{2}{*}{ Subjetivo } & $\begin{array}{l}\text { Sentido basado en el juicio-subjetivo, depende } \\
\text { de actitudes, sentimientos o puntos de vista. }\end{array}$ & $\begin{array}{l}\text { Ontología simple como realidad creada en rela- } \\
\text { ción con la función y los intereses (¿para qué } \\
\text { sirve?). También respecto de las condiciones } \\
\text { del sujeto (el dolor, por ejemplo). }\end{array}$ \\
\hline
\end{tabular}

\section{Epistemológico/ ontológico}

Objetivo Enunciados epistémicamente objetivos sobre entidades ontológicamente subjetivas.

Subjetivo Enunciados epistémicamente subjetivos sobre entidades ontológicamente objetivas.

Fuente: elaboración propia derivada de los argumentos de Searle (1997).

En el caso de la contabilidad, son innumerables los hechos sociales que responderían al criterio de ontología simple. Por ejemplo, según García (2002), el modelo económico analizado en relación con la actividad económica y los fines que persiguen los sujetos económicos que dirigen el sistema (p. 72); igual los fines de los propietarios en relación con la obtención de rentabilidad, o también la máxima u óptima obtención de productos en un proceso productivo con ocasión de la aplicación interesada de criterios de eficiencia.

Los inventarios en un proceso productivo, como otro ejemplo, pueden ser observados por la contabilidad en su forma de ontología simple y ontología compleja. Como tales, los inventarios tienen una disposición de calidad, textura, estructura física, medida, peso (ontología compleja, 
es decir, desapareciendo la función). En tanto los inventarios son observados en relación con la función que cumplen en el proceso de producción: con la finalidad de adicionar valor; formación de valor para la acumulación (ontología simple).

Un asunto importante en la distinción de los hechos institucionales, sociales o contables, se relaciona con el lenguaje. De hecho, se le critica a J. Searle su clasificación de hechos brutos e institucionales, pues, en la práctica, los hechos brutos al nombrarse a través del lenguaje realmente se convierten en institucionales. De facto, el lenguaje es igualmente un hecho institucional, pues sin este hubiese sido imposible registrar las asociaciones mentales dispuestas para la acción con sentido individual y colectivo.

Se trae a colación lo del lenguaje porque a través de este se precisan los sentidos epistemológicos y ontológicos de los objetos dispuestos en el mundo. Por lo regular, el sentido epistemológico de la distinción objetivo-subjetivo se basa en el juicio. Este juicio puede ser objetivo o subjetivo, dependiendo de los referentes del observador; según Searle (1997), los juicios subjetivos "dependen de ciertas actitudes, sentimientos o puntos de vista de los proferidores o de los oyentes del juicio en cuestión" (p. 27).

Con los juicios objetivos no solo podemos hablar de ellos como tales, sino realmente de hechos objetivos. En los juicios objetivos, según Searle (1997), "los hechos en el mundo que los convierten en verdaderos o falsos son independientes de las actitudes” (p. 27). En este sentido, en el caso de la contabilidad, los hechos contables corresponden a hechos objetivos. En palabras del profesor Moisés García (2002),

se considera hecho contable a cualquier fenómeno que altere la acumulación económica de una esfera jurídica de control patrimonial, por consiguiente, son hechos contables, tanto las operaciones económicas como los hechos económicos e incluso operaciones formales de cálculo contable. (p. 92)

Por supuesto, los hechos contables se convierten en hechos subjetivos cuando, como tales, dependen preferentemente de las actitudes o apreciaciones personales de los sujetos o agentes en cuestión. Generalmente los hechos contables como hechos subjetivos no son el resultado de los hechos económicos, los cuales, según García (2002), "son acontecimientos ajenos a la voluntad de los sujetos económicos que también impulsan la circulación económica” (p. 74).

En la versión de hechos contables-subjetivos puede encontrarse, por ejemplo, la influencia que ejerce un estilo de dirección (gerencia) en la eficiencia de obtención de los resultados o beneficios económicos de una empresa. Desde luego, el hecho contable-subjetivo lo será como hecho institucional en cuanto requiera de instituciones humanas para su reconocimiento, precise su función y esté en relación sistemática con otros hechos, o de lo contrario simplemente será un asunto eventual. Regularmente, la contabilidad tiene problemas para reconocer los hechos contables-subjetivos, en la medida en que se dificulta precisar su reconocimiento y registro.

Incluso se podría asegurar la inexistencia de hechos contables-subjetivos, en la medida en que, como tales, no puedan reconocerse a través de procesos de medición o valoración, difícilmente puede llegar a cuantificarse; aun existiendo la posibilidad de su descripción cualitativa a través de narrativas sobre su relación con las estructuras de empresa, de control o de registro. Posiblemente, el desarrollo teórico sobre la inmaterialidad e intangibilidad pueda ayudar a constituir los elementos básicos para considerar adecuadamente los hechos contables-subjetivos. 


\section{La asignación de función}

J. Searle (1997) identifica tres elementos sustanciales para dar cuenta de la realidad, en el marco de una ontología científica global: $i$ ) la asignación de función, ii) la intencionalidad colectiva, iii) las reglas constitutivas. El autor adiciona una más en el sentido de las capacidades para lidiar con el entorno.

Respecto de la asignación de función, en esta básicamente se descubre la intencionalidad, asunto que precisamente convierte a los hechos en institucionales. J. Searle (1997) afirma que en el caso de algunos artefactos, construimos el objeto para que sirva a una función: sillas, bañeras, ordenadores, ejemplos obvios. Desde este punto de vista es posible afirmar que la contabilidad es fundamental y esencialmente una disciplina de funciones, por dos razones:

1. Porque su estructura de observación, conceptual y teórica, particularmente parte de la observación de objetos que en la realidad cumplen funciones determinadas por la sociedad. Los inventarios, por ejemplo, que como cuentas de valores sirven para rendir cuentas; el dinero, que en la sociedad del intercambio emergió para facilitar la medición de contenidos; los valores de uso que en la práctica cumplen una función asignada: herramientas de trabajo, instrumentos de apoyo a la actividad, maquinaria para la operación de transformación. Es decir, una contabilidad que retoma la información apoyada en la medición y representación de objetos que cumplen una función social específica.

La importancia de los objetos en la vida social cambia dependiendo del nivel de desarrollo de la organización. Hoy, por ejemplo, los ordenadores cumplen funciones complejas respecto del procesamiento de la información, asunto que desplaza a objetos que en el pasado cumplieron una función elemental de servicio a la información, como por ejemplo los ábacos artesanales en la función de ayuda a la sumatoria y control de cuentas.

2. Porque, en sí misma, la contabilidad cumple una función de servir de apoyo a la información, entrega y rendición de las cuentas en una organización. En este sentido, la contabilidad se comporta como un objeto que se orienta hacia fines y funciones demarcadas por la sociedad y especialmente por el sistema económico y su ordenamiento. En la práctica, la contabilidad cumple una función demandada por la sociedad.

Por supuesto, la asignación de la función en los objetos no se reconocería si no mediara una intencionalidad colectiva. En este sentido, de acuerdo con Searle (1997), "las funciones nunca son intrínsecas a la física de ningún fenómeno, sino que son externamente asignadas por observadores y usuarios conscientes, en una palabra, las funciones nunca son intrínsecas sino relativas al observador" (p. 33). En el caso de la contabilidad, según Archel (2007), sus funciones se han complejizado dependiendo de intencionalidades e intereses asociados con las organizaciones, tantos que se le reconoce sus ramificaciones asociadas con estos aspectos (p. 50).

De esta manera, las funciones resultan construidas por la sociedad (observadores); por ejemplo, en el proceso de trabajo, las herramientas se constituyen en una extensión de la mano o 
del cerebro. En la praxis, el hombre/mujer hace conciencia de la función, para luego construir objetos que reemplazan funciones de la mano, el cerebro (los ordenadores, por ejemplo), u otras específicas como las de comunicación a través de la información. En este sentido, en el caso de la contabilidad, lo que la hace social no son los hechos brutos, sino el observador (la colectividad) que la convierte en hecho social e institucional.

La intencionalidad colectiva es importante de cara a los hechos institucionales porque asigna función a los objetos. Por supuesto, esto no es asunto de discrecionalidad individual, sino que, como afirma Searle (1997), tiene que ver con intereses de los usuarios y de los observadores [...], siempre que la función X es hacer $\mathrm{Y}, \mathrm{X}$ e $\mathrm{Y}$, son parte de un sistema definido generalmente por propósitos, objetivos y valores (p. 37). De esta manera es posible explicar, por ejemplo, el problema de las convenciones contables, que como tales cumplen una función, dependiendo de la colectividad, de los observadores, de los intereses y de los usuarios orientados por objetivos y valores.

\section{Sobre las convenciones contables}

Respecto de las convenciones contables, el profesor Sunder (2005) señala que muchos de los conceptos empleados por la contabilidad fueron simplemente sancionados como tales por los organismos profesionales. Por ejemplo, "los métodos contables oficialmente sancionados, en cambio, son llamados los principios contables”. En 1971, el Accounting Principles Board (APB) enumeró "los conceptos que frecuentemente son citados como convenciones (entidad contable, continuidad, periodo de tiempo, unidad monetaria, precio de intercambio)" (APB, 1971, p. 221).

De las convenciones contables es pertinente señalar que han sido el resultado de fuerzas institucionales que se encargaron de su aval social. En este ordenamiento, curiosamente, han confluido los organismos contables privados de Estados Unidos: American Institute of Certified Public Accountants (AICPA), International Accounting Standards Board (IASB) y las organizaciones públicas Securities and Exchange Commission (SEC), Organización de las Naciones Unidas (ONU), que desde tiempos pretéritos elaboraron decisiones conjugando lo teórico de la contabilidad con el poder institucional.

En las declaraciones del APB, en el año de 1964, según Tua (1983), se enunciaba taxativamente: "1. Los principios de contabilidad generalmente aceptados son aquellos que tengan apoyo de autoridad competente; 2 . las declaraciones del APB tienen apoyo de autoridad competente; 3. el apoyo de autoridad competente puede existir para otros principios contables que difieran de la opinión del APB” (p. 85); lo importante es que, como declaraciones, en la práctica han construido realidad social, pues se comprende que gran parte de las realizaciones de la contabilidad reposan en la presencia de principios denominados como de general aceptación y que corresponde a formas semánticas que cumplen el objetivo de uniformizar el espacio de comprensión de las decisiones especialmente económico-financieras.

Por supuesto, desde estas épocas de los años sesenta, los llamados principios de contabilidad generalmente aceptados instalaron la convención contable como regla más que la teoría como orientación. Desde esos años, las controversias no se han dejado esperar, según Tua (1983), como resultado de "poner de manifiesto claras diferencias entre sus deducciones teóricas y los hábitos contables del momento" (p. 89), situación que precisamente ha dado pie para reconocer en la 
contabilidad y la profesión una especie de ideología, según Eagleton (2008), caracterizada como transición del "sistema de ideas" a la ideología como una práctica social habitual y vivida (p. 220). Estas controversias se manifiestan en los contextos específicos de actuación como resultado de prácticas contables que no encuadran en la lógica de las postuladas por el modelo teórico.

Desde luego, buena parte de las explicaciones sobre el desarrollo teórico de la contabilidad y de la base sustentada de las convenciones contables forma parte de la madurez de la disciplina. Siendo la contabilidad una ciencia aplicada, se entiende que su progreso decididamente es lento, en tanto sus estructuras y acciones deben validarse por la sociedad. Las convenciones contables como tales, en este sentido, forman parte de la validación social y de las reglas de uso de las sociedades empresariales. Podría afirmarse que, a un menor número de convenciones contables, posiblemente una mayor validez científica, asunto que indicaría un cierto grado de madurez disciplinaria.

Desde el marco de las convenciones puede resultar menos costoso y más rápido resolver problemas que por otra vía puede ser hasta imposible. Parte del éxito de la convención contable tiene que ver con su inserción en la cultura, lo cual facilita su difusión, aunque precisamente se lesione el desarrollo teórico.

En el anterior sentido, los principios de contabilidad generalmente aceptados (PCGA) son convención porque cumplen la función de minimizar riesgos económicos; si se incrementan los riesgos, disminuye el beneficio económico. Desde luego, las convenciones contables también pueden ser explicadas desde un marco ideológico. Precisamente la ideología, en una de sus versiones, sustenta que los enunciados pueden simplemente traducirse en un conjunto de creencias orientadas a la acción. De tal manera, las convenciones contables podrían solo referirse a pactos institucionales para garantizar las acciones en determinados sentidos.

Según Sunder (2005, p. 221), para que un modelo de comportamiento sea una convención, debe satisfacerse ciertas condiciones: $i)$ debe aplicarse a condiciones recurrentes y no solo a una clase de eventos, ii) tiene que ser de conocimiento común, iii) es del interés de todos que una persona más se ajuste a la convención (p. 222). Mencionado por este autor, "Gilmer identificó dos características de las proposiciones contables que constituyen una convención: 1) están basadas en el acuerdo general, y 2) son establecidas más o menos arbitrariamente" (p. 222).

Independiente de la manera como la convención contable se inserte en el medio (por imposición de los operadores, por ejemplo) está atravesada por el lenguaje. Desde Searle (1997) puede decirse que es el lenguaje, los símbolos, las palabras que significan o expresan en su relación con la comprensión pública, las que configuran la convención contable, determinándola como hecho institucional.

De facto, la intencionalidad colectiva (que no necesariamente involucra a toda la sociedad) le otorga funciones de estatus a las cosas, no siendo diferente para el caso de la contabilidad. Por supuesto, las convenciones contables se entienden como acuerdos realizados por agentes con intereses especialmente económicos, los cuales determinan su uso y aplicación. En este sentido, resulta pertinente la apreciación de J. Habermas (2010), quien afirma:

Mientras que la validez de las reglas técnicas y de las estrategias depende de la validez de enunciados empíricamente verdaderos o analíticamente correctos, la validez de las normas sociales solo se funda en la intersubjetividad del acuerdo sobre intenciones y solo viene asegurada por el reconocimiento general de las obligaciones. (p. 21) 
Un aspecto importante en la determinación de convenciones contables es el interés económico del agente (operador, administrador, propietario), quien en últimas lo determina con su proceder. Por ejemplo, el concepto de entidad contable es vital porque de este dependen los poderes del propietario o del capital, incluso el(los) tipo(s) de control(es) implementado(s); según Sunder (2005), "puesto que tiene realmente consecuencias económicas, la elección de entidad no es arbitraria y por consiguiente no es una cuestión de convención” (p. 225).

Es indiscutible la presencia de tensiones relacionadas con los operadores de la contabilidad, que puede entenderse como la tensión entre la convención contable y el cuerpo explicativo teórico contable. La Financial Accounting Standards Board (FASB), por ejemplo, fracasa en su intento de establecer normas contables basadas en los principios soportados en la estructura de un marco conceptual y, más que ello, en su imposición; según Sunder (2005) "cuando la FASB propuso a los bancos reconocer la pérdida del valor económico de los préstamos [...], los bancos asumieron una posición unificada contra la propuesta" (p. 102).

En otras palabras, las convenciones contables lo son en la medida en que no afecten los intereses económicos de los agentes o la estructura económica de la contabilidad. La aplicación de la partida doble, en su sentido de equilibrio entre los pasivos y los activos, puede corresponder a una convención en cuanto la sumatoria de los registros no afecta intereses económicos. Sin embargo, es posible identificar que esta forma de proceder, según Miller y O’leary (1987), se explica mejor si se comprende "una relación más o menos directa entre los intereses políticos y económicos, y los conocimientos y técnicas que los representan” (p. 129).

La importancia del convencionalismo contable emerge en relación con las prácticas contables, que en general se enmarcan en la diversidad de referentes y ejercicios de acción. Definitivamente, las prácticas contables se desarrollan en contextos específicos de actuación organizacional y empresarial. Por este motivo, resulta difícil el ejercicio de comparabilidad y uniformidad de las cifras contables. Podría incluso afirmarse que cuando se habla de comparación y uniformización, realmente se está ante la presencia de un intento por el lenguaje de convención que propugne por la emergencia de criterios para permitir el logro de la homogeneización universal de las prácticas contables que, como se afirma, resultan contextuadas.

En últimas, esta especial convención afincada en la comparación y la uniformidad, tal vez, no tenga otro propósito que facilitar el camino y presencia del derecho contable comparado, en la vía de la resolución de problemáticas formuladas por la transnacionalización de los negocios y del capital.

El respeto por las convenciones contables resultantes de las prácticas contables y de gestión, específicas y contextuadas, adscritas a modos socioeconómicos de producción, puede consistir en la alternativa que marque un camino de construcción económica diferente al conocido como modelo económico imperante basado en el individualismo. Por supuesto, el asunto tendrá que ver con la constitución de referentes teóricos fuertes, apalancados en una ciencia normativa que logre vincular lo general con lo específico de las actuaciones y diferencias.

Desde luego, el imperio de las convenciones contables aprobadas por los organismos reguladores internacionales es igualmente el paso hacia la construcción de formalidades que permitan validar lo adecuado o no en la aplicación de las normativas. La FASB, con sus desarrollos apoyados en principios como derivaciones del trabajo pasado, alrededor del APB, coloca la enunciación de 
las políticas contables como una práctica convencional, que se supone debe garantizar el conocimiento de las formas y reglas de confección y aplicación de los principios y procedimientos para la obtención de la información contable. Según Miller y O’leary (1987), este tipo de formalidades, son garantía de un trabajo que permita establecer las variaciones dadas respecto de la autoridad competente (p. 204).

Las convenciones contables se asocian al discurso de la contabilidad, en cuanto generan sentidos con alta probabilidad de construir verdades. Tal vez, en este aspecto esté centrado el enorme poder social de la convención. Al comprenderse como discurso, la convención adquiere sentido. De allí, las convenciones contables, en la práctica, construyen realidades, que menos tienen que ver con verdades derivadas de estructuras lógico-semánticas y más con lenguajes sociales que construyen sentidos en relación con la aceptación o el rechazo.

De acuerdo con lo anterior, las convenciones ponen en tensión el sentido y criterio de la epistemología y de las reglas para derivar conocimientos (verdaderos o falsos). En la perspectiva de Searle (1997), las convenciones contables, vistas desde la función, realmente cumplen con objetivos y propósitos. Decir, por ejemplo, que "las cifras de los estados financieros se han obtenido de acuerdo con los principios de contabilidad generalmente aceptados" es reafirmar el propósito de legitimación de la autoridad competente que emite los principios; o decir que las cifras corresponden con la "imagen fiel y verdadera", es afirmar que las cifras han sido preparadas de acuerdo con normas de contabilidad. En última instancia, se trataría de construcciones que corresponden a un punto de vista ontológico de la realidad como creación subjetiva; definitivamente se trata de "hechos sociales" y como tales subjetivos.

Desde luego, la contabilidad comunica, y como tal está unida a lenguajes, discursos, códigos en presencia, signos y señales. Los códigos, de alguna manera, han demarcado referentes, los cuales, como afirma Lee (2006a y 2006b) interpretando a Macintosh, se transforman en "signos que relacionan la contabilidad con el referente".

En la evolución histórica de la contabilidad, progresivamente se reconoce que cambian los referentes. Estos se han complejizado poco a poco, transportando una nueva concepción social que se desplaza de lo material a lo inmaterial. En las actuales sociedades, las relaciones entre las cosas y los objetos se constituyen en un nuevo referente; tal vez importe más nombrar las relaciones que las cosas u objetos mismos. Por ejemplo, la moneda como referente de relación ha cambiado de simple punto de referencia a convertirse en medio de pago (nuevo referente). Estos referentes corresponden a construcciones sociales que impelen comprensiones o emergencias de "nuevas realidades". Lo importante aquí es que muchos de los referentes de la contabilidad realmente se han convertido en convenciones contables.

Desde la perspectiva de Searle, se puede afirmar que las convenciones contables se constituyen en verdaderas fuentes de hechos institucionales. De hecho, las convenciones contables cumplen con finalidades derivadas del lenguaje, el acuerdo y la función. Como tales, las convenciones no resultan independientes de creencias, valores e ideología y en general del sentido de los observadores. En la práctica, las convenciones contables construyen realidad, y en esa medida forman parte de los acuerdos, no solo de la sociedad, sino también de la comunidad correspondiente que las interpreta y usa. 
Las convenciones contables (tabla 3), como hechos institucionales, requieren de instituciones como el mercado, las transacciones, la información; incluso requieren de una comunidad que las interpreta y usa. Por supuesto, y siguiendo a Searle (1997), las convenciones contables no solo existen por fuera de un marco institucional que las legitima, sino en relación con otras convenciones y hechos que en conjunto forman relaciones sistemáticas. En la práctica, según Searle (1997), las convenciones contables se instalan "merced al acuerdo o la aceptación colectiva" (p. 56) que las valida dependiendo de los que se sirven de ellas.

Tabla 3. Algunos ejemplos de convención contable

\begin{tabular}{|c|c|}
\hline Convención & Explicación \\
\hline Entidad contable & $\begin{array}{l}\text { No es una convención en cuanto afecta la estructura económica } \\
\text { de los agentes. }\end{array}$ \\
\hline Comparabilidad y uniformidad & $\begin{array}{l}\text { Convención que cumple la finalidad de coadyuvar el acuerdo; no } \\
\text { afecta la estructura económica de la contabilidad. }\end{array}$ \\
\hline $\begin{array}{l}\text { Los estados financieros se han elabo- } \\
\text { rado de acuerdo con los PCGA }\end{array}$ & Es convención en cuanto enuncia un acuerdo. \\
\hline Periodo contable & $\begin{array}{l}\text { Es convención. Generalmente no afecta la estructura económica } \\
\text { del agente. }\end{array}$ \\
\hline Número; valores expresados en dinero & Es convención, por el dinero que es de uso social común. \\
\hline Sistema contable & Convención en cuanto solo cumple un papel analítico. \\
\hline
\end{tabular}

Fuente: elaboración del autor.

\section{Conclusiones}

Buena parte de los senderos de la investigación contable tendrán que ver con las respuestas sobre la realidad en la contabilidad: ¿`cómo la contabilidad concibe la realidad? Algunas aproximaciones pueden obtenerse desde Jhon Searle, aunque con las reservas propias de lo que significa la búsqueda de nuevos sentidos y comprensiones sobre el trabajo que hace y debe realizar la contabilidad.

El principal aporte, desde las apreciaciones de Searle (1997), tiene que ver con lo denominado como hechos institucionales, los cuales requieren para su identificación del cumplimiento de requisitos, siendo la "función" el principal de ellos, determinados por la presencia de la intencionalidad colectiva y el acuerdo.

En el sentido del trabajo de Searle (1997), es posible identificar a la contabilidad como un campo de conocimiento enteramente funcional que se orienta hacia el cumplimiento de fines específicos. Tal vez sea posible, igualmente, identificar a la contabilidad como uno de los hechos institucionales más destacados de la historia de las representaciones sociales. En este sentido, por supuesto, formaría parte de la historia de la modernidad que logró constituir tres referentes destacados: el mercado, el marco institucional y la moneda. La contabilidad, en este escenario, como 
lo afirma J. Searle de los hechos institucionales, cumpliría funciones especiales, no solo para posibilitar su empoderamiento como hecho, sino igualmente para construir nuevos hechos institucionales, como nuevas realidades.

Un aspecto sugerente de Searle (1997) está relacionado con su clasificación de los hechos: brutos e institucionales. Para Searle, los hechos brutos forman parte de la identificación o descripción de estos en su estructura material o composición de elementales. Los hechos institucionales, como tales, se identifican por estar asociados a la presencia de valores, creencias, cogniciones, actitudes e intencionalidades grupales, donde se destaca el papel vinculante del observador. En este sentido, referimos que la contabilidad, como cuerpo de conocimientos, (en la versión de Searle) ha tenido que vérsela realmente con hechos fundamentalmente institucionales. Al respecto es posible arriesgar la sugerencia de que los hechos brutos, siendo como tales para la contabilidad, en la práctica lo sean como institucionales para otra disciplina del conocimiento (la física, por ejemplo).

Tal vez lo más importante de la postura de Searle (1997) sea la comprensión que realiza de los hechos institucionales en relación con las intencionalidades grupales o colectivas. En el caso de la disciplina contable, se puede identificar claramente la presencia de los reguladores públicos y privados, que, desligados del marco de la validez científica, postulan normativas bajo criterios imperativos de "autoridad reconocida y competente", para su cabal cumplimiento. Estos reguladores realmente son reconocidos por su intencionalidad grupal, manifiesta en los pronunciamientos contables. Por la vía de la intencionalidad, incluso se han validado asuntos de la contabilidad que tienen menos que ver con las prácticas científicas y más con las convenciones, en el entendido que las convenciones, como tales, igualmente se constituyen en hechos institucionales destacados.

$\mathrm{Si}$ se identifica a la contabilidad como un campo de conocimientos funcional que responde a intereses específicos, y que se orienta básicamente hacia el mantenimiento, producción y reproducción de convenciones contables, posiblemente se esté formulando la presencia de una disciplina deontológica, que valore la moralidad no en función de los resultados, sino de la obediencia categórica de determinados principios orientadores de la acción. Se tratará, entonces, de la contabilidad como un hecho institucionalizado, funcional y apoyado en prácticas de acción soportadas en la convención.

El examen juicioso de la contabilidad y de sus prácticas lleva a reconocer que muchas de sus postulaciones son realmente convenciones. Se habla, por ejemplo, de Sistemas contables en presencia, cuando estos realmente constituyen sentidos con diferentes y variados órdenes de organización, dependiendo de los criterios empresariales. Para algunos tratadistas, como otro ejemplo, el concepto de entidad es una forma de organización que, por la vía institucional-jurídica, permite establecer los límites de las responsabilidades y de los derechos, con la finalidad de proteger rendimientos, asunto que se convierte en convencional. Desde luego, para otros el concepto de entidad no es convencional en cuanto afecta las estructuras económicas de la contabilidad.

La indagación de los hechos institucionales en relación con las convenciones contables da pie, muy seguramente, para precisar el camino epistemológico y ontológico de la contabilidad. De alguna manera, la epistemología como filosofía de la ciencia ha derivado en condicionantes, requisitos y cánones de postulación y desarrollo, que corresponden a una vía de fundamentación de las ciencias. Desde esta perspectiva, la epistemología contable tendría el enorme trabajo 
de fundamentar la ciencia contable, asunto de naturaleza científica que puede conducir hacia la refundación de un campo de conocimiento, que no necesariamente tenga que ver con las exigencias sociales y económicas bajo las cuales se postulan las transacciones que operan en contextos diversos.

Desde el ángulo ontológico de la contabilidad, esta disciplina puede ser más proclive a las especificaciones contextuales de actuación y, mucho más útil en el relacionamiento con los agentes económicos y las necesidades de los múltiples usuarios de la información. Por supuesto, lo objetivo y lo subjetivo siempre estará presente, y más cuando se establece la estricta separación entre objeto y sujeto, asunto que, como tal, también es susceptible de interpretarse como la gran convención de la ciencia de Occidente.

\section{Referencias}

2. Abbagnano, N. (2007). Diccionario de filosofía. México: Fondo de Cultura Económica.

3. Archel, P. (2007). Teoría e investigación en contabilidad. Un caso de estudio. Madrid: AECA.

4. Berger, P. \& Luckmann, T. (2001). La construcción social de la realidad. Buenos Aires.

5. Eagleton, T. (2008). La ideología y sus vicisitudes en el marxismo occidental. En S. Zizek (coord.), Ideología un mapa de la cuestión (pp. 199-253). Buenos Aires: Fondo de Cultura Económica.

6. Gaffikin, M. (2006). The Critique of accounting theory. Faculty of Commerce Accounting \& Finance Working Papers, 41, 1-21. Recuperado de http://ro.uow.edu.au/accfinwp/41

7. García, M. (2002). Contabilidad y circulación económica. Una visión nueva y unificada de la contabilidad. Madrid: Pearson Educación.

8. Habermas, J. (1999). Teoría de la acción comunicativa. 2: critica de la razón funcionalista. Madrid: Taurus.

9. Habermas, J. (2010). Ciencia y técnica como ideología. Madrid: Tecno.

10. Hendriksen, E. S. (1974). Teoría de la contabilidad. México: Uteha.

11. Laughlin, R. (1999). Critical accounting: The nature, progress and prognosis of Accounting. Auditing and Accountability Journal, 12 (1), 73-78.

12. Lee, T. (2006a). The FASB and accounting for economic reality. Accounting and the Public Interest, 6, 1-27.
13. Lee, T. (2006b). Reply to commentary —Cunning plans, spinners, and ideologues: Blackadder and Baldick try accounting for economic reality. Accounting and the Public Interest, 6, 45-50.

14. Luhmann, N. (1998). Sistemas sociales. Lineamientos para una teoría general. Barcelona: Anthropos.

15. Miller P. \& O'leary T. (1987/2009). La contabilidad y la construcción de la persona gobernable. En M. Gómez \& C. Ospina, Avances interdisciplinarios para una comprensión critica de la contabilidad (pp. 127-163). Bogotá, D. C.: Universidad Nacional de Colombia y Universidad de Antioquia.

16. Nicolás J. A. \& Frápolli, M. J. (2012). Teorías contemporáneas de la verdad. Madrid: Tecnos.

17. Searle, J. (1997). La construcción de la realidad social. Barcelona: Editorial Paidós.

18. Searle, J. (2006). ¿Qué es una institución? Revista de Derecho Politico (UNED), 66, 89-120.

19. Simmel, G. (2010). Cultura líquida y dinero: Fragmentos simmelianos de la Modernidad. México: Anthropos.

20. Sunder, S. (2005). Teoría de la contabilidad y el control. Bogotá, D. C.: Universidad Nacional de Colombia.

21. Tua, J. (1983). Principios y normas de contabilidad. España: Instituto de Planificación Contable, Ministerio de Economía y Hacienda. 